\title{
Pruning Severity in High Density Guava for Higher Returns
}

\author{
Pankaj Nautiyal $^{1 *}$, Shant Lal ${ }^{2}$, Gaurav Papnai ${ }^{1}$, Neeraj Joshi ${ }^{1}$ and Varun Supyal ${ }^{1}$ \\ ${ }^{1}$ Krishi Vigyan Kendra, ICAR-VPKAS, Chinyalisaur-249146, Distt Uttarkashi, \\ Uttarakhand, India \\ ${ }^{2}$ Department of Horticulture, G B Pant University of Agriculture \& Technology, \\ Pantnagar 263145, Uttarakhand, India \\ *Corresponding author
}

\section{A B S T R A C T}

\section{Keywords}

Guava, High density, Pruning severity, Nonfruiting shoots, Winter season

\section{Article Info}

Accepted:

18 August 2020 Available Online: 10 September 2020
The present experiment was carried out at Horticulture Research Centre, Patharchatta, Department of Horticulture, GBPUA\&T, Pantnagar, India under high density planting of guava cv. Pant Prabhat. The study was conducted to find out the interaction effect of pruning severity and plant spacing on growth, yield and profit. The treatments consisted of two plant spacing and seven pruning severity viz., One leaf pair shoot pruning $\left(\mathrm{P}_{1}\right.$, OLP:); Two leaf pairs shoot pruning $\left(\mathrm{P}_{2}, \mathrm{TLP}\right)$; Three leaf pairs shoot pruning $\left(\mathrm{P}_{3}\right.$, THLP); Thinning out of non-fruiting shoots + One leaf pair shoot pruning $\left(\mathrm{P}_{4}\right.$, TNFS+OLP); Thinning out of non-fruiting shoots + Two leaf pairs shoot pruning $\left(\mathrm{P}_{5}\right.$, TNFS+TLP); Thinning out of non-fruiting shoots + Three leaf pairs shoot pruning $\left(\mathrm{P}_{6}\right.$, TNFS+THLP); Thinning out of non-fruiting shoots only $\left(\mathrm{P}_{7}\right.$, TNFS) and no pruning $\left(\mathrm{P}_{0}\right)$ as control. In this way there were eight treatments with sixteen combinations replicated four times each with two plants in one experimental unit. The annual increase in plant height, plant spread and plant volume were found maximum in unpruned plants $\left(\mathrm{P}_{0}\right)$, while, the stem diameter was found maximum with treatment $\mathrm{P}_{4}$ (TNFS+OLP). The pruning severity had significantly decreased the per cent fruit set for rainy season crop and subsequently increased the per cent fruit set for winter season crop. The higher fruit yield was obtained with treatment $\mathrm{P}_{4}$ (TNFS+OLP) followed by treatment $\mathrm{P}_{1}(\mathrm{OLP})$. The unpruned plants $\left(\mathrm{P}_{\mathrm{O}}\right)$ produced lowest fruit yield in winter season. The highest benefit: cost ratio was calculated with the treatment $\mathrm{P}_{4}$ (TNFS+OLP) and $2.0 \times 1.0 \mathrm{~m}$ plant spacing. Hence, for achieving higher winter season crop with maximum profit under high density plantation of guava, complete removal of non-fruiting shoots followed by one leaf pair shoot pruning of current seasons growth should be done during first week of May.

\section{Introduction}

Guava (Psidium guajava L.) is famous as "poor man's fruit" and belongs to family
Myrtaceae (Prakash et al., 2002). Guava occupies $5^{\text {th }}$ position in terms of area after mango, citrus, banana and apple and also $5^{\text {th }}$ position in production in India. It contributes 
3.90 percent of the total production of fruits in India, which is around 4.236 million tonnes from an area of 0.276 thousand hectare and productivity is around 15.35 tonnes per hectare (NHB, 2019).

It has a very important fruit due to its nutrient content and is known as "The apple of tropics". Guava is the third richest source of vitamin C (200-300 mg/100g of pulp), after Barbados cherry (Malpighia emarginata) (1000-4000 mg/100 g pulp) and Aonla (600 $\mathrm{mg} / 100 \mathrm{~g}$ pulp) fruit (Gupta and Naik, 2008). Besides vitamin $\mathrm{C}$, guava fruits are also rich source of vitamin A (250 IU/100g fruit pulp), pectin $(0.5-1.8 \%)$, riboflavin $\left(\mathrm{B}_{2}, 0.02-0.04\right.$ $\mathrm{mg} / 100 \mathrm{~g}$ pulp), and thiamine ( $\mathrm{B}_{1}, 0.03-0.07$ $\mathrm{mg} / 100 \mathrm{~g}$ pulp) as compared to other fruits (Sharma, 2010; Pal et al., 2017). In tropical and subtropical conditions of northern India, two major flowering seasons are common i.e., summer (April-May) and rainy (JulyAugust). The summer (April- May) flowering produces rainy season crop and rainy season (July- August) flowering produces winter season crop. In tarai conditions of India, guava trees produce $85-90 \%$ crop in rainy season and 8-10\% in winter season. This feature in guava cultivation is a major source of concern. Rainy season fruits are rough, insipid, poor in quality, less nutritive, easily affected by insect pests and have very short shelf life and due to these characteristics the guava fruits do not fetch remunerative prices. Whereas, the winter season fruits are superior in quality, free from diseases and pests and fetch more prices in the market. Winter season crop have better storage life and thus can be transported to destination offering remunerative prices (Singh et al., 2001, Tyagi and Patel, 2004; Tiwari and Lal, 2007).

Guava is a pruning responsive crop and fruiting occurs in current season growth. Shoot pruning in high density orchards is prerequisite to maintain the desired canopy of this fast growing guava plant (Lal et al., 2000). The efficient training and pruning can maintain the proper canopy size of the guava tree, improve fruit quality and provide opportunity to increase the number of trees per unit area (Nautiyal et al., 2016). Presently shoot pruning has emerged as eco-friendly alternative method for regulating the guava crop. It is free from all the demerits of existing methods. On the other hand, pruning may be helpful in reducing the tree size and improving the fruit quality (Lal et al., 2007). Shoot pruning in high density orchards is prerequisite to maintain the desired canopy of this fast growing guava plant. The need exists for some refinement in shoot pruning technique where flowering and fruit setting can be regulated to attain higher winter season fruiting especially for high density plantation.

\section{Materials and Methods}

The study was conducted at Horticulture Research Centre, Patharchatta, Department of Horticulture, G. B. Pant University of Agriculture and Technology, Pantnagar, India. Five years old grafted, well trained guava trees of uniform growth (modified leader system) and planted under high density were selected for the study. The treatments consisted of two plant spacing (i.e. $2.0 \mathrm{~m} \mathrm{x}$ $1.0 \mathrm{~m}$ and $2 \mathrm{~m} \times 2 \mathrm{~m}$ spacing) and seven pruning severity [One leaf pair shoot pruning $\left(\mathrm{P}_{1}\right.$, OLP:); Two leaf pairs shoot pruning $\left(\mathrm{P}_{2}\right.$, TLP); Three leaf pairs shoot pruning $\left(\mathrm{P}_{3}\right.$, THLP); Thinning out of non-fruiting shoots + One leaf pair shoot pruning $\left(\mathrm{P}_{4}, \mathrm{TNFS}+\mathrm{OLP}\right)$; Thinning out of non-fruiting shoots + Two leaf pairs shoot pruning $\left(\mathrm{P}_{5}\right.$, TNFS+TLP); Thinning out of non-fruiting shoots + Three leaf pairs shoot pruning $\left(\mathrm{P}_{6}\right.$, TNFS+THLP); Thinning out of non-fruiting shoots $\left(\mathrm{P}_{7}\right.$, TNFS). with no pruning $\left(\mathrm{P}_{0}\right)$ as control (Plate 1). In this way there were eight treatments with sixteen combinations replicated four times each with two plants in one 
experimental unit. The experiment was laid out in factorial randomized block design. During the month of February, in the first year, all the plants were topped to a uniform height of $1.75 \mathrm{~m}$ from the ground level. Emergence of new shoot began after 20-25 days of topping. The same procedure was followed in the month of February next year. Shoot pruning of current season's growth was done as per treatment, with the help of secateurs, in the last week of April during both the years of study. The observations on growth and yield attributes and economic analysis were recorded during both the seasons (Rainy and winter season). The data were analyzed according to the procedure of analysis for Factorial Randomized Block Design. The significance of variation among the treatments was observed by applying ' $\mathrm{F}$ ' test and critical difference at 5 per cent probability was calculated to compare the mean values of treatments for all the characters.

\section{Results and Discussion}

\section{Effect of pruning severity and plant spacing on plant growth}

The interaction between plant spacing and pruning severity, non-significantly affected the plant height (Table 1). However, the maximum annual increment in plant height was observed with treatment combination $\mathrm{S}_{2}$ $\mathrm{P}_{0}(2.0 \times 2.0 \mathrm{~m}$ plant spacing and unpruned plants) during both the years. While minimum increment in plant height was found with treatment combination $\mathrm{S}_{1} \mathrm{P}_{4}$ (plant spaced at $2.0 \times 1.0 \mathrm{~m}$ and TNFS+ OLP) during first and second year. The interaction of plant spacing and pruning severity gave significant effect on plant spread during both the years (Table 1). The maximum increment in plant spread was observed with treatment combination $\mathrm{S}_{2} \mathrm{P}_{0}(2.0 \times 2.0 \mathrm{~m}$ plant spacing and unpruned plants) during both the years. The annual increase in plant height and plant spread were found maximum in unpruned plants $\left(\mathrm{P}_{0}\right)$. As pruning severity increased, decrease in plant height and plant spread was observed.

The data presented in Table 2 shows that interaction among plant spacing and pruning severity non-significantly affected the stem diameter during both the years. The maximum annual increment in stem diameter was observed with treatment combination $\mathrm{S}_{2} \mathrm{P}_{4}$ (2.0x $2.0 \mathrm{~m}$ and TNFS+OLP) during both the years. The interaction between plant spacing and pruning severity non-significantly affected the plant volume (Table 2). The higher annual increase in plant volume was observed with treatment combination $\mathrm{S}_{2} \mathrm{P}_{0}$ (plant spacing $2.0 \times 2.0 \mathrm{~m}$ and unpruned plant) during both the years. The lowest annual increment in plant volume was observed with treatment combination $\mathrm{S}_{1} \mathrm{P}_{4}$ (plant spacing $2.0 \times 1.0 \mathrm{~m}$ and TNFS+OLP).

Therefore, data pertaining to plant growth suggested that wider spacing $(2.0 \times 2.0 \mathrm{~m})$ gave higher increase in plant height, plant spread, stem diameter and plant volume. As spacing increased the plant growth increases significantly. It was due to more light penetration in wider spacing and subsequently accumulation of more photosynthetic reserves in plants. The annual increase in plant growth was recorded minimum in closed spacing due to less light penetration into foliage and restricted apical growth, ultimately reducing plant growth and vigour. While working in different plant spacing of guava crop, similar reports have also been reported (Lal et al., 2007; Pilania et al., 2010; Pratibha et al., 2013; Pal and Lal, 2015). The annual increase in plant height, plant spread and plant volume were found maximum in unpruned plants $\left(\mathrm{P}_{0}\right)$ while the stem diameter was found maximum with treatment $\mathrm{P}_{4}$ (TNFS+OLP) As pruning severity increased, decrease in plant growth except stem diameter was observed. The 
shoot pruning severity overcomes apical dominance thus, increasing the activity of cytokinine which helps to promote growth in terms of stem diameter. In other hand, the stored carbohydrates in plants and available nutrients in soil have been utilized by plants, which might have been used for further growth of pruned shoots (Marini, 2014). Therefore, the severely pruned plants showed minimum annual increment in plant growth.

\section{Effect of pruning severity and spacing on yield attributes}

The data presented in Table 3 unveil that the severity of pruning and plant spacing significantly affected per cent fruit set for both the seasons (rainy and winter) during both the years. Among the plant spacing, 2.0 $\times 2.0 \mathrm{~m}$ plant spacing $\left(\mathrm{S}_{2}\right)$ gave higher per cent fruit set $(34.30 \%$ and $35.49 \%)$ for rainy season crop during both the years, while, plant spaced at $2.0 \times 1.0 \mathrm{~m}\left(\mathrm{~S}_{1}\right)$ gave maximum per cent fruit set $(72.95 \%$ and $71.24 \%$ ) for winter season crop during both the years.

The pruning severity had significantly decreased the per cent fruit set for rainy season crop during both the years and subsequently increased the per cent fruit set for winter season crop during both the years. The higher per cent fruit set $(65.55 \%$ and $71.17 \%$ ) was recorded with unpruned plants $\left(\mathrm{P}_{\mathrm{O}}\right)$ for rainy season crops followed by treatment $\mathrm{P}_{7}$ (TNFS). The minimum fruit set percentage (14.82 \% and $15.42 \%)$ was obtained with treatment $\mathrm{P}_{4}$ (TNFS+OLP) in rainy season crop during both the years. However, for winter season crop, the maximum per cent fruit set $(77.76 \%$ and $78.05 \%$ ) was recorded with treatment $\mathrm{P}_{4}$ (TNFS+OLP) followed by treatment $\mathrm{P}_{6}$ (TNFS+THLP). The minimum fruit set percentage (59.61\% and $66.08 \%$ ) was recorded in treatment $\mathrm{P}_{7}$ (TNFS) during both the years. The data presented in Table 4 clearly showed that severity of pruning had significant effect on per cent fruit retention for both the seasons during both the years. The highest fruit retention percentage $(51.03 \%$ and $45.08 \%)$ was recorded in unpruned plants $\left(\mathrm{P}_{0}\right)$, while, lowest $(9.18 \%$ and $9.12 \%$ ) was observed with treatment $\mathrm{P}_{4}$ (TNFS+OLP) in rainy season crop during both the years. However, in winter season the highest fruit retention percentage $(53.64 \%)$ was recorded with treatment $\mathrm{P}_{3}$ (THLP) in the first year of experiment. During second year, the maximum per cent fruit retention was obtained in treatment $\mathrm{P}_{6}$ (TNFS+THLP) followed by treatment $\mathrm{P}_{4}$ (TNFS with OLP) in winter season crop.

A critical observation of data presented in Table 5 clearly indicates that pruning severity, plant spacing and their interaction (Fig 1) significantly affected the yield per hectare in both seasons (rainy and winter) during both the years of experiment. The highest fruit yield (q) per hectare (204.97 q/ha and 187.37 $\mathrm{q} / \mathrm{ha}$ ) was obtained with unpruned plants. While, lowest fruit yield (q) per hectare (43.35 q/ha and $40.54 \mathrm{q} / \mathrm{ha}$ ) was obtained with treatment $\mathrm{P}_{4}$ (TNFS+OLP) in rainy season during both the years. However, the reverse trend was observed in winter season crop. The higher fruit yield (q/ha) (207.71 and 189.50 $\mathrm{q} / \mathrm{ha}$ ) was obtained with treatment $\mathrm{P}_{4}$ (TNFS+OLP) followed by treatment $\mathrm{P}_{1}$ (OLP). The unpruned plants $\left(\mathrm{P}_{\mathrm{O}}\right)$ produced lowest fruit yield (41.19 and $36.32 \mathrm{q} / \mathrm{ha})$ in winter season during both the years of investigation.

It was observed that as increased the plant population, the fruit yield (q) per hectare also increased. The highest total annual yield (q) per hectare (251.06 and $230.04 \mathrm{q} / \mathrm{ha})$ was found with treatment $\mathrm{P}_{4}$ (TNFS+OLP) followed by unpruned plants $\left(\mathrm{P}_{\mathrm{O}}\right)$ during both the year. However, the lowest total annual 
yield (q) per hectare was obtained in treatment $\mathrm{P}_{6}$ (TNFS+ THLP) in first year and $\mathrm{P}_{2}$ (TLP) in the second year of experiment. Among the spacing, the plant spaced at $2.0 \times$ $1.0 \mathrm{~m}\left(\mathrm{~S}_{1}\right)$ produced higher yield (q) per hectare in both seasons (i.e. rainy and winter) during both the years of experiment. These results are in accordance with the earlier findings (Tiwari and Lal, 2007; Pratibha et al., 2013; Joshi et al., 2014; Kumawat et al., 2014; Thakre et al., 2016; Joshi et al., 2016; Nautiyal et al., 2016).

Pruning severity with complete removal of non fruiting shoots significantly decreased the yield in rainy season crop and subsequently increased the yield in winter season crop. This type of trend might be due to response of plant hormones, stored carbohydrates and available nutrients in soil. Shoot pruning overcomes the apical dominance and increases the activity of cytokinin and gibberellins, which promotes emergence of new shoots. Cytokinin content and its activity is very high in all growing shoots of pruned plants (Murray, 2010). Elevated growth of new vegetation occurs after pruning. It strongly reduces nutrient reserves, in particular of carbohydrates, from unpruned plant parts (Marini, 2014). Shoot pruning, thus, affects the physiological processes of photosynthesis and non-structural carbohydrate synthesis. Non-structural carbohydrate reserves, mainly starch provide the energy to drive re-growth of pruned plants for rainy season flowering (i.e. winter season fruiting).The stored carbohydrates in plants and available nutrients in soil, which might have been used for further growth of nonfruiting shoots, have been utilized for emergence of new shoots and subsequently for impressive morphometric growth and enhanced fruit quality of winter season crop (Bagachi et al., 2008).

Table.1 Interaction effect of plant spacing and pruning severity on plant height and spread of guava cv. Pant Prabhat

\begin{tabular}{|c|c|c|c|c|c|c|c|c|c|}
\hline \multirow[t]{3}{*}{ Treatments } & \multirow{3}{*}{$\frac{\infty}{8}$} & \multicolumn{4}{|c|}{$\begin{array}{l}\text { Annual increase in plant height } \\
\text { (m) }\end{array}$} & \multicolumn{4}{|c|}{$\begin{array}{c}\text { Annual increase in plant } \\
\text { spread (m) }\end{array}$} \\
\hline & & \multicolumn{2}{|c|}{ First year } & \multicolumn{2}{|c|}{ Second year } & \multicolumn{2}{|c|}{ First year } & \multicolumn{2}{|c|}{ Second year } \\
\hline & & $\mathbf{S}_{1} *$ & $\mathbf{S}_{2} *$ & $\mathbf{S}_{1}$ & $\mathbf{S}_{2}$ & $\mathbf{S}_{1}$ & $\mathbf{S}_{2}$ & $\mathbf{S}_{1}$ & $\mathbf{S}_{2}$ \\
\hline Unpruned & $\mathrm{P} 0$ & 1.723 & 1.803 & 1.640 & 1.780 & 1.148 & 1.203 & 1.098 & 1.145 \\
\hline OLP & $\mathrm{P} 1$ & 1.485 & 1.573 & 1.523 & 1.433 & 0.928 & 0.983 & 0.870 & 0.965 \\
\hline TLP & $\mathrm{P} 2$ & 1.535 & 1.598 & 1.463 & 1.500 & 0.963 & 0.980 & 0.888 & 0.933 \\
\hline THLP & P3 & 1.638 & 1.715 & 1.483 & 1.653 & 0.935 & 1.015 & 0.885 & 0.958 \\
\hline TNFS+OLP & $\mathrm{P} 4$ & 1.448 & 1.485 & 1.395 & 1.425 & 0.883 & 0.913 & 0.865 & 0.898 \\
\hline TNFS+ TLP & P5 & 1.525 & 1.543 & 1.420 & 1.448 & 0.960 & 0.973 & 0.870 & 0.900 \\
\hline $\begin{array}{l}\text { TNFS+ } \\
\text { THLP }\end{array}$ & P6 & 1.543 & 1.598 & 1.428 & 1.485 & 0.945 & 0.955 & 0.940 & 0.893 \\
\hline TNFS & P7 & 1.648 & 1.708 & 1.470 & 1.635 & 1.013 & 1.155 & 0.960 & 1.015 \\
\hline SEm \pm & & \multicolumn{2}{|c|}{0.019} & \multicolumn{2}{|c|}{0.042} & \multicolumn{2}{|c|}{0.029} & \multicolumn{2}{|c|}{0.038} \\
\hline CD at $5 \%$ & & \multicolumn{2}{|c|}{ NS } & \multicolumn{2}{|c|}{ NS } & \multicolumn{2}{|c|}{ NS } & \multicolumn{2}{|c|}{ NS } \\
\hline CV (\%) & & \multicolumn{2}{|c|}{2.430} & \multicolumn{2}{|c|}{5.690} & \multicolumn{2}{|c|}{5.900} & \multicolumn{2}{|c|}{8.085} \\
\hline
\end{tabular}

$*\left(\mathrm{~S}_{1}-2.0 \times 1.0 \mathrm{~m}, \mathrm{~S}_{2}-2.0 \times 2.0 \mathrm{~m}\right)$ 
Table.2 Interaction effect of plant spacing and pruning severity on stem diameter and plant volume of guava cv. Pant Prabhat

\begin{tabular}{|c|c|c|c|c|c|c|c|c|c|}
\hline \multirow[t]{3}{*}{ Treatments } & \multirow{3}{*}{$\begin{array}{l}\frac{n}{8} \\
\text { है } \\
\text { है }\end{array}$} & \multicolumn{4}{|c|}{$\begin{array}{l}\text { Annual increase in stem diameter } \\
\qquad(\mathrm{cm})\end{array}$} & \multicolumn{4}{|c|}{$\begin{array}{c}\text { Annual increase in plant } \\
\text { volume }\left(\mathrm{m}^{3}\right)\end{array}$} \\
\hline & & \multicolumn{2}{|c|}{ First year } & \multicolumn{2}{|c|}{ Second year } & \multicolumn{2}{|c|}{ First year } & \multicolumn{2}{|c|}{ Second year } \\
\hline & & $\mathbf{S}_{1}^{*}$ & $\mathbf{S}_{2} *$ & $S_{1}$ & $\mathbf{S}_{2}$ & $\mathbf{S}_{1}$ & $\mathbf{S}_{2}$ & $S_{1}$ & $\mathbf{S}_{2}$ \\
\hline Unpruned & P0 & 0.858 & 1.098 & 0.923 & 1.125 & 1.185 & 1.361 & 1.032 & 1.221 \\
\hline OLP & $\mathrm{P} 1$ & 1.360 & 1.610 & 1.383 & 1.653 & 0.667 & 0.796 & 0.549 & 0.700 \\
\hline TLP & P2 & 1.255 & 1.425 & 1.278 & 1.535 & 0.748 & 0.803 & 0.604 & 0.689 \\
\hline THLP & P3 & 1.155 & 1.305 & 1.213 & 1.380 & 0.747 & 0.925 & 0.607 & 0.794 \\
\hline TNFS+OLP & P4 & 1.335 & 1.740 & 1.368 & 1.743 & 0.589 & 0.647 & 0.603 & 0.601 \\
\hline TNFS+ TLP & P5 & 1.390 & 1.710 & 1.348 & 1.698 & 0.734 & 0.762 & 0.562 & 0.614 \\
\hline $\begin{array}{l}\text { TNFS+ } \\
\text { THLP }\end{array}$ & P6 & 1.218 & 1.543 & 1.310 & 1.653 & 0.726 & 0.764 & 0.664 & 0.625 \\
\hline TNFS & P7 & 1.083 & 1.170 & 1.163 & 1.245 & 0.882 & 1.191 & 0.711 & 0.880 \\
\hline SEm \pm & & \multicolumn{2}{|c|}{0.0615} & \multicolumn{2}{|c|}{0.0544} & \multicolumn{2}{|c|}{0.0493} & \multicolumn{2}{|c|}{0.0572} \\
\hline CD at $5 \%$ & & \multicolumn{2}{|c|}{ NS } & \multicolumn{2}{|c|}{ NS } & \multicolumn{2}{|c|}{ NS } & \multicolumn{2}{|c|}{ NS } \\
\hline CV (\%) & & \multicolumn{2}{|c|}{9.309} & \multicolumn{2}{|c|}{7.910} & \multicolumn{2}{|c|}{11.677} & \multicolumn{2}{|c|}{15.991} \\
\hline
\end{tabular}

Table.3 Effect of plant spacing and pruning severity on per cent fruit set of guava cv. Pant Prabhat

\begin{tabular}{|c|c|c|c|c|c|}
\hline \multirow{3}{*}{ Treatments } & \multirow{3}{*}{$\begin{array}{l}\frac{n}{8} \\
\text { हो } \\
\text { हो }\end{array}$} & \multicolumn{4}{|c|}{ Fruit set (\%) } \\
\hline & & \multicolumn{2}{|c|}{ First year } & \multicolumn{2}{|c|}{ Second year } \\
\hline & & Rainy season & Winter season & Rainy season & Winter season \\
\hline \multicolumn{6}{|l|}{ Spacing } \\
\hline $2.0 \times 1.0 \mathrm{~m}$ & $\mathrm{~S}_{1}$ & $34.46(35.28)$ & 72.95(58.97) & $35.21(35.76)$ & $71.29(57.73)$ \\
\hline $2.0 \times 2.0 \mathrm{~m}$ & $\mathrm{~S}_{2}$ & $34.30(35.25)$ & 69.57(56.70) & $35.49(36.02)$ & 70.06(56.92) \\
\hline \multicolumn{2}{|l|}{ SEm \pm} & $(0.40)$ & $(0.90)$ & $(0.33)$ & $(0.52)$ \\
\hline \multicolumn{2}{|l|}{ CD at 5\% } & $(1.52)$ & (3.38) & $(0.93)$ & (1.48) \\
\hline \multicolumn{6}{|c|}{ Pruning severity } \\
\hline Unpruned & P0 & $65.66(54.14)$ & 70.95(57.45) & 71.17(57.56) & 67.99(55.59) \\
\hline OLP & P1 & $14.82(22.61)$ & $70.45(57.59)$ & $15.05(22.78)$ & 68.49(55.92) \\
\hline TLP & P2 & $24.38(29.54)$ & 71.61(57.93) & $25.18(30.10)$ & 71.38(57.74) \\
\hline THLP & P3 & $35.93(36.81)$ & $72.26(58.51)$ & $36.62(37.21)$ & 71.86(58.04) \\
\hline TNFS+OLP & P4 & $14.82(22.59)$ & 77.76(61.90) & $15.42(23.06)$ & $78.05(62.20)$ \\
\hline TNFS+ TLP & P5 & $22.68(28.34)$ & 70.78(57.40) & $23.15(28.70)$ & 68.92(56.16) \\
\hline TNFS+ THLP & P6 & $33.93(35.58)$ & 76.63(61.25) & $33.77(35.52)$ & $72.63(58.53)$ \\
\hline TNFS & P7 & $62.84(52.47)$ & $59.61(50.65)$ & $62.46(52.22)$ & $66.08(54.42)$ \\
\hline \multicolumn{2}{|l|}{ SEm \pm} & $(0.80)$ & (1.78) & $(0.65)$ & (1.04) \\
\hline \multicolumn{2}{|l|}{ CD at $5 \%$} & (3.04) & $(6.77)$ & $(1.85)$ & $(2.97)$ \\
\hline \multicolumn{2}{|l|}{ CV $(\%)$} & $(6.42)$ & $(8.71)$ & (5.13) & $(5.15)$ \\
\hline
\end{tabular}

\#Figures in parenthesis indicate transformed value 
Table.4 Effect of plant spacing and pruning severity on per cent fruit retention of guava cv. Pant Prabhat

\begin{tabular}{|c|c|c|c|c|c|}
\hline \multirow{3}{*}{ Treatments } & \multirow{3}{*}{ 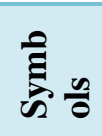 } & \multicolumn{4}{|c|}{ Fruit retention (\%) } \\
\hline & & \multicolumn{2}{|c|}{ First year } & \multicolumn{2}{|c|}{ Second year } \\
\hline & & Rainy season & Winter season & Rainy season & Winter season \\
\hline \multicolumn{6}{|l|}{ Spacing } \\
\hline $2.0 \times 1.0 \mathrm{~m}$ & $\mathrm{~S}_{1}$ & $17.80(24.08)$ & $45.98(42.66)$ & $17.01(23.60)$ & $44.96(42.08)$ \\
\hline $2.0 \times 2.0 \mathrm{~m}$ & $\mathrm{~S}_{2}$ & $23.70(28.29)$ & $55.03(47.93)$ & $22.80(27.77)$ & $52.00(46.16)$ \\
\hline \multicolumn{2}{|l|}{ SEm \pm} & $(0.35)$ & $(0.85)$ & $(0.34)$ & $(0.58)$ \\
\hline \multicolumn{2}{|l|}{ CD at $5 \%$} & NS & NS & NS & NS \\
\hline \multicolumn{6}{|l|}{ Pruning severity } \\
\hline Unpruned & P0 & $51.03(45.60)$ & $42.66(40.75)$ & $45.08(42.15)$ & $40.85(39.70)$ \\
\hline OLP & $\mathrm{P} 1$ & $11.98(20.10)$ & $53.38(46.95)$ & $10.61(18.91)$ & $47.27(43.43)$ \\
\hline TLP & P2 & $15.91(23.43)$ & $52.45(46.46)$ & $14.85(22.60)$ & $48.21(43.98)$ \\
\hline THLP & P3 & $19.95(26.42)$ & $53.64(47.09)$ & $21.14(27.29)$ & $49.77(44.85)$ \\
\hline TNFS+OLP & P4 & $9.18(17.54)$ & $52.94(46.71)$ & $9.12(17.42)$ & $53.53(47.05)$ \\
\hline TNFS+ TLP & P5 & $12.01(20.20)$ & $53.19(46.84)$ & $13.19(21.18)$ & $51.55(45.89)$ \\
\hline TNFS+ THLP & P6 & $14.19(22.05)$ & $52.52(46.52)$ & $14.72(22.43)$ & $55.96(48.47)$ \\
\hline TNFS & P7 & $31.5(34.17)$ & $43.28(41.07)$ & $30.54(33.42)$ & $40.68(39.60)$ \\
\hline \multicolumn{2}{|l|}{ SEm \pm} & $(0.70)$ & $(1.70)$ & $(0.68)$ & (1.16) \\
\hline \multicolumn{2}{|c|}{ CD at $5 \%$} & (1.99) & $(4.84)$ & (1.95) & (3.33) \\
\hline \multicolumn{2}{|c|}{ CV $(\%)$} & $(7.55)$ & (10.62) & $(7.56)$ & (7.49) \\
\hline
\end{tabular}

\#Figures in parenthesis indicate transformed value

Table.5 Effect of plant spacing and pruning severity on yield (q/ha) of guava cv. Pant Prabhat

\begin{tabular}{|c|c|c|c|c|c|c|c|}
\hline \multirow{3}{*}{ Treatments } & \multirow{3}{*}{ है } & \multicolumn{6}{|c|}{ Yield (q/ha) } \\
\hline & & \multicolumn{3}{|c|}{ First year } & \multicolumn{3}{|c|}{ Second year } \\
\hline & & $\begin{array}{l}\text { Rainy } \\
\text { season }\end{array}$ & $\begin{array}{l}\text { Winter } \\
\text { season }\end{array}$ & $\begin{array}{l}\text { Total } \\
\text { yield }\end{array}$ & $\begin{array}{l}\text { Rainy } \\
\text { season }\end{array}$ & $\begin{array}{l}\text { Winter } \\
\text { season }\end{array}$ & $\begin{array}{l}\text { Total } \\
\text { yield }\end{array}$ \\
\hline \multicolumn{8}{|l|}{ Spacing } \\
\hline $2.0 \times 1.0 \mathrm{~m}$ & $\mathrm{~S}_{1}$ & 99.94 & 143.45 & 243.39 & 97.13 & 137.48 & 234.69 \\
\hline $2.0 \times 2.0 \mathrm{~m}$ & $\mathrm{~S}_{2}$ & 73.30 & 103.64 & 176.94 & 71.13 & 91.87 & 163.00 \\
\hline \multicolumn{2}{|c|}{ SEm \pm} & 3.96 & 2.32 & 3.24 & 1.95 & 2.02 & 2.82 \\
\hline \multicolumn{2}{|c|}{ CD at $5 \%$} & NS & NS & NS & NS & NS & $\mathrm{NS}$ \\
\hline \multicolumn{8}{|c|}{ Pruning severity } \\
\hline Unpruned & P0 & 204.97 & 41.19 & 246.16 & 187.37 & 36.32 & 223.69 \\
\hline OLP & $\mathrm{P} 1$ & 51.88 & 171.68 & 223.56 & 43.97 & 149.27 & 193.24 \\
\hline TLP & $\mathrm{P} 2$ & 67.69 & 122.57 & 190.27 & 63.37 & 113.85 & 177.21 \\
\hline THLP & P3 & 89.40 & 102.63 & 192.03 & 92.19 & 94.30 & 186.49 \\
\hline TNFS+OLP & $\mathrm{P} 4$ & 43.35 & 207.71 & 251.06 & 40.54 & 189.50 & 230.04 \\
\hline TNFS+ TLP & P5 & 53.16 & 153.71 & 206.87 & 57.44 & 143.12 & 200.56 \\
\hline TNFS+ THLI & P6 & 61.46 & 118.25 & 179.71 & 64.20 & 124.01 & 188.21 \\
\hline TNFS & P7 & 121.06 & 70.61 & 191.67 & 123.98 & 67.05 & 191.03 \\
\hline \multicolumn{2}{|c|}{ SEm \pm} & 3.96 & 4.65 & 6.48 & 3.90 & 4.05 & 5.66 \\
\hline \multicolumn{2}{|c|}{ CD at $5 \%$} & 11.29 & 13.27 & 18.46 & 11.12 & 11.56 & 16.13 \\
\hline \multicolumn{2}{|c|}{ CV (\%) } & 12.94 & 10.66 & 8.72 & 13.12 & 10.01 & 8.06 \\
\hline
\end{tabular}


Table.6 Total cost of production (per hectare) of high density planting of guava cv. Pant Prabhat planted at $2.0 \mathrm{x} 1.0 \mathrm{~m}$ spacing $\left(\mathrm{S}_{1}\right)$ (average of both the years)

\begin{tabular}{|c|c|c|c|c|c|c|c|c|}
\hline E⿱艹⿹勹巳 & Abbreviation & $\begin{array}{c}\text { Operational cost } \\
\text { ha/year (Rs.) } \\
\text { [A] }\end{array}$ & $\begin{array}{c}\text { Input cost (Rs.) } \\
{[\mathrm{B}]}\end{array}$ & $\begin{array}{c}\text { Packaging and } \\
\text { Transportation } \\
\text { charge (Rs.) } \\
{[\mathrm{C}]}\end{array}$ & $\begin{array}{c}\text { Total cost of } \\
\text { production/ha/year } \\
(\text { Rs. }) \\
{[D=A+B+C]}\end{array}$ & $\begin{array}{c}\text { Treatment } \\
\text { application } \\
\text { cost/plant/year } \\
\text { (Rs.) }\end{array}$ & $\begin{array}{c}\text { Treatment } \\
\text { application } \\
\text { Cost/ ha^/year } \\
\text { (Rs.) }[\mathrm{X}]\end{array}$ & $\begin{array}{c}\text { Total cost/ha/year } \\
(\text { Rs. }) \\
(\mathrm{E}=\mathrm{X}+\mathrm{D})\end{array}$ \\
\hline $\mathbf{P}_{1}$ & OLP & 83250.00 & 42280.00 & 13000.00 & 138530.00 & $4.80^{*}$ & 24000.00 & 162530.00 \\
\hline $\mathbf{P}_{2}$ & TLP & 83250.00 & 42280.00 & 13000.00 & 138530.00 & $4.80 *$ & 24000.00 & 162530.00 \\
\hline $\mathbf{P}_{3}$ & THLP & 83250.00 & 42280.00 & 13000.00 & 138530.00 & $4.80 *$ & 24000.00 & 162530.00 \\
\hline $\mathbf{P}_{4}$ & TNFS+OLP & 83250.00 & 42280.00 & 13000.00 & 138530.00 & $6.25^{\#}$ & 31250.00 & 169780.00 \\
\hline $\mathbf{P}_{5}$ & TNFS+TLP & 83250.00 & 42280.00 & 13000.00 & 138530.00 & $6.25^{\#}$ & 31250.00 & 169780.00 \\
\hline $\mathbf{P}_{6}$ & TNFS+THLP & 83250.00 & 42280.00 & 13000.00 & 138530.00 & $6.25^{\#}$ & 31250.00 & 169780.00 \\
\hline $\mathbf{P}_{7}$ & TNFS & 83250.00 & 42280.00 & 13000.00 & 138530.00 & $3.85^{\# \#}$ & 19250.00 & 157780.00 \\
\hline
\end{tabular}

(*52 plants/labour/day; ${ }^{\#} 40$ plants/labour/day; ${ }^{\#} 65$ plants/labour/day; Labour cost @ 250/day; ^ 5000 plants/ha])

Table.7 Economic analysis of high density planting of guava cv. Pant Prabhat planted at 2.0 x $1.0 \mathrm{~m} \mathrm{spacing}\left(\mathrm{S}_{1}\right)$ (average of both the years)

\begin{tabular}{|c|c|c|c|c|c|c|c|c|c|c|}
\hline \multirow[b]{2}{*}{ 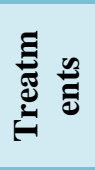 } & \multirow[b]{2}{*}{$\frac{\dot{0}}{2}$} & \multirow{2}{*}{$\begin{array}{c}\text { Total cost of } \\
\text { production/ha/y } \\
\text { ear (Rs.) }\end{array}$} & \multicolumn{3}{|c|}{ Avg. Yield (q/ha) } & \multicolumn{3}{|c|}{ Gross return/ha/year (₹) } & \multirow{2}{*}{$\begin{array}{c}\text { Net } \\
\text { return/ha/yea } \\
\text { r (Rs.) }\end{array}$} & \multirow{2}{*}{$\begin{array}{c}\text { Benefit: } \\
\text { cost } \\
\text { ratio }\end{array}$} \\
\hline & & & Rainy & Winter & Total & $\begin{array}{c}\text { Rainy } \\
\left(\begin{array}{l}@ \\
\text { Rs. 12/kg) }\end{array}\right.\end{array}$ & $\begin{array}{l}\text { Winter (@ } \\
\text { Rs.20/kg) }\end{array}$ & $\begin{array}{l}\text { Total } \\
\text { (Rs.) }\end{array}$ & & \\
\hline $\mathbf{P}_{\mathbf{0}}$ & Unpruned & 138530.00 & 233.04 & 43.99 & 277.03 & 279648.00 & 87980 & 367628.00 & 215648.00 & 1.56 \\
\hline $\mathbf{P}_{1}$ & OLP & 162530.00 & 52.87 & 192.21 & 245.08 & 63444.00 & 384420 & 447864.00 & 268134.00 & 1.65 \\
\hline $\mathbf{P}_{2}$ & TLP & 162530.00 & 76.22 & 137.93 & 214.16 & 91464.00 & 275860 & 367324.00 & 187594.00 & 1.15 \\
\hline $\mathbf{P}_{3}$ & THLP & 162530.00 & 104.91 & 114.45 & 219.36 & 125892.00 & 228900 & 354792.00 & 175062.00 & 1.08 \\
\hline $\mathbf{P}_{4}$ & TNFS+OLP & 169780.00 & 45.33 & 237.74 & 283.07 & 54396.00 & 475480 & 529876.00 & 342146.00 & 2.02 \\
\hline $\mathbf{P}_{5}$ & TNFS+TLP & 169780.00 & 65.89 & 173.29 & 239.19 & 79068.00 & 346580 & 425648.00 & 237918.00 & 1.40 \\
\hline $\mathbf{P}_{6}$ & TNFS+THLP & 169780.00 & 74.81 & 141.58 & 216.39 & 89772.00 & 283160 & 372932.00 & 185202.00 & 1.09 \\
\hline $\mathbf{P}_{7}$ & TNFS & 157780.00 & 135.23 & 82.57 & 217.81 & 162276.00 & 165140 & 327416.00 & 152686.00 & 0.97 \\
\hline
\end{tabular}

(\#Treatments abbreviations:- $\mathrm{P}_{1}$, OLP: One leaf pair shoot pruning; $\mathrm{P}_{2}$, TLP: Two leaf pairs shoot pruning; $\mathrm{P}_{3}$, THLP: Three leaf pairs shoot pruning; $\mathrm{P}_{4}$ TNFS+OLP: Thinning out of non-fruiting shoots + One leaf pair shoot pruning; $\mathrm{P}_{5}$, TNFS+TLP: Thinning out of non-fruiting shoots + Two leaf pairs shoot pruning; $\mathrm{P}_{6}$, TNFS+THLP: Thinning out of non-fruiting shoots + Three leaf pairs shoot pruning; $\mathrm{P}_{7}$, TNFS: Thinning out of non-fruiting shoots). 
Int.J.Curr.Microbiol.App.Sci (2020) 9(9): 2704-2716
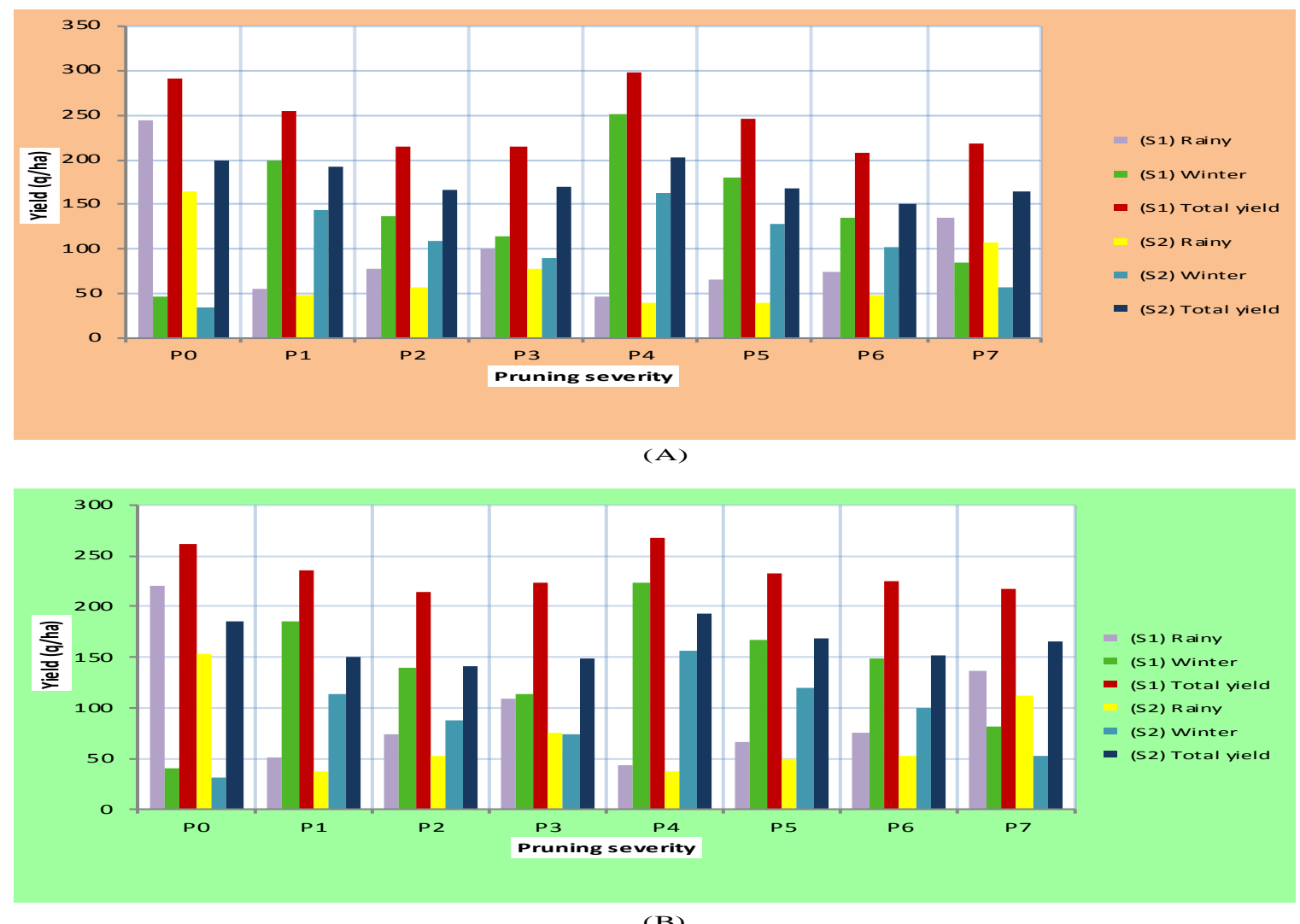

(B)

Fig 1 : Interaction effect of plant spacing and pruningse ve rity on yield (q/ha) of guava cv. Pant Prabhat during the years (A) $2014-15$ and (B) $2015-16$. 


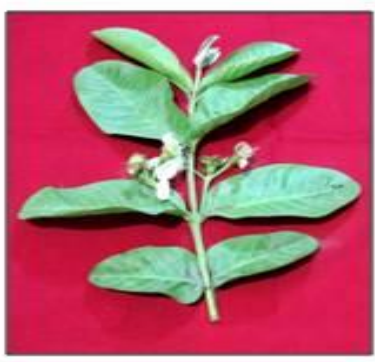

$\mathbf{P e}$

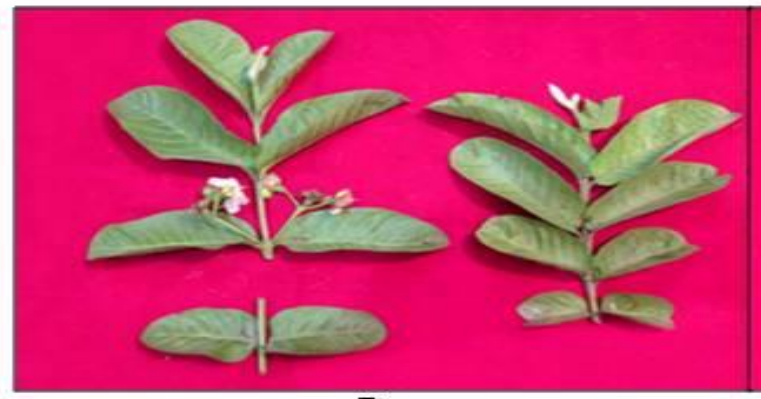

$\mathbf{P}$

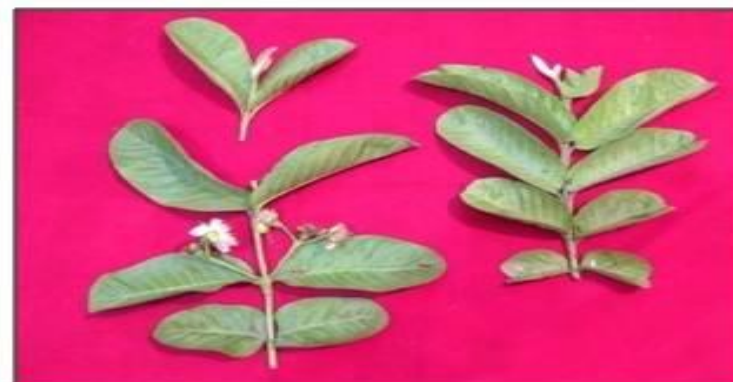

$\mathbf{P}_{\mathbf{E}}$

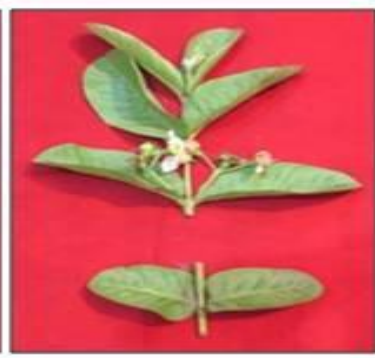

P1

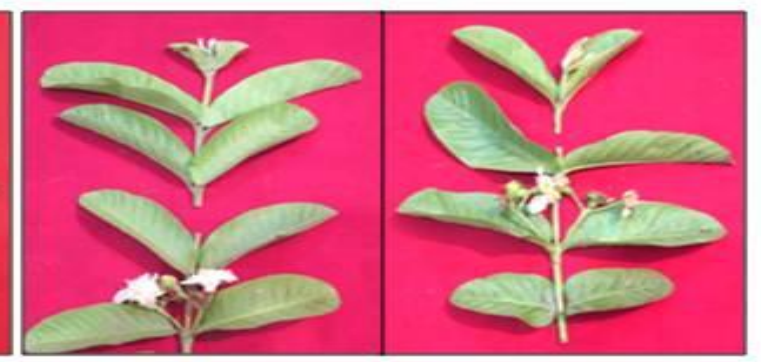

$\mathbf{P}_{\mathbf{z}}$

$\mathbf{P}_{3}$

$\mathbf{P}_{0}=$ Unpruned; $\mathbf{P}_{1}$, OL.P: One Laf pair sheot pruning; $\mathbf{P}_{2}$, TLP: Twe Laf pairs sheet

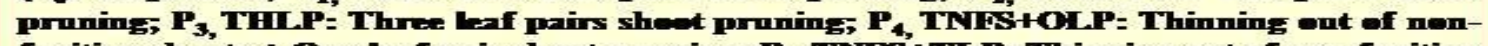
fruiting shems + One leaf pair sheet pruning; $P_{5}$, TNifs + TL.P: Thinning ont of non-firuiting shows + Twe Laf pairs sheot pruning; $P_{G}$, TNifs+THLP: Thinning out of non-firuiting sheots + Three leaf pairs shemt pruning; $P_{7}$, TNFS: Thinning out of non-firuiting shemts) Plate 4 : Details of the treatments 


\section{Economic analysis of experiment}

The cost of production per hectare per year (average of both the years) presented in the Table 6. The highest total cost of production (per hectare) of high density planting of guava planted at $2.0 \times 1.0 \mathrm{~m}$ spacing $\left(\mathrm{S}_{1}\right)$ occurred Rs. $1,69,780.00$ per ha per year with three treatments, $\mathrm{P}_{4}$ (TNFS+OLP), $\mathrm{P}_{5}$ (TNFS+TLP) and $\mathrm{P}_{6}$ (TNFS+THLP). The cost of initial inputs (cost of fertilizers, irrigation water, machinery, chemicals and labour cost etc.) were equal to all treatments i.e. Rs. $1,38,530.00$ per hectare per year. The treatment cost of unpruned plants $\left(\mathrm{P}_{0}\right)$ was nil due to untreated plants.

The maximum gross return and net return per hectare per year (Table 7) in close spaced plants $(2.0 \times 1.0 \mathrm{~m}$ plant spacing $)$ were recorded with treatment $\mathrm{P}_{4}$ (TNFS+OLP) followed by $\mathrm{P}_{1}(\mathrm{OLP})$. While, minimum gross return and net return per hectare per year was obtained with treatment $\mathrm{P}_{7}$ (TNFS). The maximum benefit: cost ratio (2.02:1) was obtained with treatment $\mathrm{P}_{4}$ (TNFS+OLP) followed by $\mathrm{P}_{1}$ (OLP). However, minimum benefit: cost ratio $(0.97: 1)$ was found with the treatment $\mathrm{P}_{7}$ (TNFS).

In general, it was observed that maximum benefit: cost ratio was found with the treatment $\mathrm{P}_{4}$ (TNFS+OLP) while minimum benefit: cost ratio was recorded with treatment $\mathrm{P}_{7}$ (TNFS). This might be due to treatment $\mathrm{P}_{4}$ gave highest yield during winter season which fetches higher market prices as compared to rainy season crop. Minimum benefit: cost ratio was found with treatment $\mathrm{P}_{7}$ (TNFS) might be due to the lower winter season yield as well as total yield. It was also reported that it is desirable to take a sole winter crop instead of two to three crops in a year for higher returns (Singh et al., 2000). It was observed that on the basis of yield and return during both the seasons, for regulating the cropping pattern in guava, one leaf pair shoot pruning during first week of May, was found most suitable for maximum winter season yield with superior fruit quality (Tiwari and Lal, 2007). Further it was reported that the maximum cost: benefit was obtained with one leaf pair pruning of fruited shoots only due to higher production of quality fruits during winter season (Thakre $e t$ al., 2016).

On the basis of above observations, it can be concluded that plant growth fruit yield and profitability of guava can be influenced by pruning severity. Fruit yield per hectare increased with increase in plant population per unit area. The maximum benefit: cost ratio was found with the treatment complete removal of non fruiting shoots followed by one leaf pair shoot pruning due to high winter season fruiting. Hence, for higher profitability in high density plantation of guava, complete removal of non fruiting shoots followed by one leaf pair shoot pruning of current season growth (i.e. retaining one leaf pair at the base of the newly emerged shoots) should be done during first week of May. This method is environmental friendly and completely organic because it has been performed without using any chemical and pruned leaves act as mulch, thereby, save water in summer months and after degradation in rainy season they are rich source of manure.

\section{References}

Bagachi T B, Sukul P and Ghosh B. 2008. Biochemical changes during off-season flowering in guava (Psidium guajava L.) induced by bending and pruning. Journal of Tropical Agriculture, 46(12): 64-66.

Gupta S N and Naik K B 2008. Instant Horticulture pp 206-7 Jain Brothers, New Delhi.

Joshi P, Lal S, Nautiyal P and Pal M. 2014. 
Response of plant spacing and pruning intensity on yield contributing characteristics of guava cv. Pant Prabhat. Journal of Hill Agriculture, 5(2): 163-167.

Joshi P, Lal S, Nautiyal P and Pal M. 2016. Effect of plant spacing and pruning intensity on flowering, fruiting, and yield of guava (Psidium guajava L.) cv. Pant Prabhat. International Journal of Agriculture Sciences, 8(7):1064-1068.

Kumawat K L, Sarolia D K, Kaushik R A and Jodha A S. 2014. Effect of different spacing on newly planted guava cv 1-49 under ultra high density planting system. African Journal of Agricultural Research, 9(51): 3729-3735.

Lal S, Tiwari J P and Misra K K. 2000. Effect of plant spacing and pruning intensity on fruit yield and quality of guava. Progressive Horticulture, 32(3-4) (1): 20-25.

Lal S, Tiwari J P, Awasthi P and Mahajan A R. 2007. Studies on planting systems in guava (Psidium guajava L.) cv. Sardar. Acta Horticulture, 735: 263-266.

Marini R. 2014. Physiology of pruning fruit trees. Virginia cooperative Extension Publication, 422-025:1-8.

Murray J. 2010. Tree hormones and why they matter. Arnoldia Arboretum, 67(4): 1519.

Nautiyal P, Lal S, and Singh C P. 2016. Effect of shoot pruning severity and plant spacing on leaf nutrient status and yield of guava cv. Pant Prabhat. International Journal of Basic and Applied Agricultural Research, 14(3):288-294.

Nautiyal P, Lal S, Dimri D C and Arora I. 2016. Shoot pruning severity in high density of guava (Psidium guajava L.). International Journal of Agriculture Sciences, 8(52):2427-2431.

NHB. 2019. NHB, Gurgaon, Haryana, http://www.nhb.gov.in/statistics/State_L evel/2018-
19\%20(3rd\%20Adv.Est_.)\%20-

$\% 20$ Website.pdf

Pal M and Lal S. 2015. Effect of different high density planting on growth and yield of guava cv. Pant Prabhat. International Journal of Basic and Applied Agricultural Research,13(3): 420-424.

Pal M, Lal S, Nautiyal P and Joshi P. 2017. Response of high density spacing on physico-chemical quality and yield of guava (Psidium guajava L.) cv. Pant Prabhat. International Journal of Agriculture Sciences, 9(9):3962-3965.

Pilania S, Shukla A K, Mahawer L N, Sharma $\mathrm{R}$ and Bairwa H L. 2010. Standardization of pruning intensity and integrated nutrient management in meadow orcharding of guava (Psidium guajava L.). Indian Journal of Agricultural Sciences, 80 (8): 673-678.

Prakash D P, Narayanaswamy P and Sondur S N. 2002. Analysis of molecular diversity in guava using RAPD markers. Journal of Horticulture Science and Biotechechnology, 77: 287-293.

Pratibha, Lal S and Goswami A K. 2013. Effect of pruning and planting systems on growth, flowering, fruiting and yield of guava (Psidium guajava L.) cv. Sardar. Indian Journal of Horticulture, 70(40):496-500.

Sharma S K. 2010. Postharvest Management and Processing of Fruits and Vegetables - Instant Notes, pp 390-92. New India Pub Agency New Delhi.

Singh G, Singh A K and Pandey D. 2000. Effect of cropping pattern on fruiting behavior of guava (Psidium guajava L.) trees. Annals of Agriculture Research. 21 (2): 175-182.

Singh G, Singh A K and Rajan S. 2001. Influence of pruning date on fruit yield of guava (Psidium guajava L.) under subtropics. Journal of Applied Horticulture. 3(1): 37-40. 
Thakre M, Lal S, Uniyal S, Goswami A K and Prkash P. 2016. Pruning for crop regulation in high density guava (Psidium guajava L.) plantation. Spanish Journal of Agriculture Research. 14(2): e0905. http://dx.doi.org/10.5424/sjar/20161427846

Tiwari J P and Lal S. 2007. Effect of NAA, flower bud thinning and pruning on crop regulation in guava (Psidium guajava L.) cv. Sardar. Acta Horticulture. 735: 311-314.

Tyagi S K and Patel R M. 2004. Effect of growth regulators on rooting of air layering of guava (Psidium guajava L.). Orissa Journal of Horticulture, 4:23-27.

\section{How to cite this article:}

Pankaj Nautiyal, Shant Lal, Gaurav Papnai, Neeraj Joshi and Varun Supyal. 2020. Pruning Severity in High Density Guava for Higher Returns. Int.J.Curr.Microbiol.App.Sci. 9(09): 27042716. doi: https://doi.org/10.20546/ijcmas.2020.909.337 